Veniard, Marie. « 'Ne pas parler à la place des premiers concernés'. Étude d'une pratique politique d'éthique langagière chez des militants politiques pour les droits des étrangers en France. » Nouvelle

\title{
"Ne pas parler à la place des premiers concernés ». Étude d'une pratique politique d'éthique langagière chez des militants politiques pour les droits des étrangers en France
}

\author{
Marie Veniard \\ Université de Paris \\ Laboratoire EDA \\ France
}

\begin{abstract}
Cet article a pour objet une pratique d'éthique langagière ancrée dans le champ des mobilisations politiques pour les droits des étrangers. II s'agit en particulier de décrire ce que nous nommerons dans un premier temps une norme interactionnelle, celle de « ne pas parler à la place des premiers concernés » tel qu'elle se réalise dans ce champ de luttes. Cette norme prend sens dans ce qu'on appelle les « luttes pour la reconnaissance » (Honneth, 2013/2018), des militantes féministes, des militants pour les droits des homosexuels, des immigrés ... Dans ce cadre, elle participe du processus de visibilisation des groupes en situation de domination (Voirol, 2005) : associée à l'idée plus générale de faire entendre sa voix, cette norme circule dans les sphères militantes (par exemple dans les luttes féministes, dans le journal féministe Le torchon brûle ${ }^{\circ} 1$, dès 1971, voir annexe 1), L. Greco (2018) le mentionne dans les luttes queer ; on la trouve aussi dans les démarches d'éducation populaire (de Lepinay, 2019). C'est une idée qui circule également dans la sphère académique, ce qui amène un renouvellement des pratiques de recherche, à l'initiative d'intellectuels engagés : pour la France, en histoire, chez G. Noiriel (Une histoire populaire de la France, 2018), en sociologie chez P. Bourdieu (La Misère du monde, 1993).
\end{abstract}

Je me limiterai pour ma part au domaine de la mobilisation de militants sans-papiers pour leurs droits d'étrangers et de militants «soutiens" pour les droits des étrangers. Ces derniers sont qualifiés de « militants par conscience » par la sociologie, car ne se mobilisant pas pour leurs propres droits et nommés "les soutiens ${ }^{1}$ » par les acteurs (j'alternerai entre ces dénominations). Les titres de quelques ouvrages d'acteurs de ces luttes témoignent de la circulation dans le champ de cette norme interactionnelle: Parole de sans-papiers (Madjiguène Cissé, 1999, récit d'une des luttes historiques de personnes immigrantes en situation irrégulière à Paris en 1996) ou Les voix de la jungle par le collectif Les Écrivains de Calais (2020) (témoignages de migrants installés dans le campement informel de Calais, France).

Lors d'observations de terrain auprès d'un collectif de soutien aux migrants et d'un collectif de sanspapiers, j'ai repéré des comportements langagiers qui relèvent, j'en fais l'hypothèse, d'une norme langagière que je nomme "Ne pas parler à la place des premiers concernés ", ci-après NP3. Je formule une seconde hypothèse: cette norme relève d'une idéologie langagière considérant la parole politique comme une voie d'émancipation possible et un lieu de pouvoir. Cette conscience réflexive du pouvoir qui peut être exercé par la parole amènerait les militantes et militants à faire de cette injonction un régime éthique qui guiderait les différentes interactions auxquelles ils sont amenés à participer. Ces interactions, observées lors de réunions (enregistrées), de rencontres informelles ou de manifestations, ainsi que dans des documents écrits circulant parmi les membres du groupe constituent mes données de terrain. Les éléments théoriques et méthodologiques seront développés dans les sections 1 et 2. Dans la section 3, je décrirai la manière dont s'actualise " Ne pas parler à la place ... » dans sa dimension pratique, d'abord à travers des gestes, puis au fil du discours, dans l'intra-discours, et enfin, dans les interactions, à travers l'analyse de règles locales telles que " $\mathrm{Ne}$ pas donner d'ordre à un premier concerné ", " $\mathrm{Ne}$ pas interrompre un premier concerné ». L'analyse des interactions est menée dans une perspective goffmanienne de figuration², intégrée à l'analyse de discours.

Mon travail est guidé par la nécessité d'envisager les productions langagières dans leur dynamique sociale, en portant l'attention sur la manifestation et la circulation des normes du groupe. Ainsi, il emprunte à différentes disciplines des sciences du langage, notamment la sociolinguistique et l'analyse des interactions. Cependant, son ancrage principal se situe en analyse de discours de tradition française, ce qui implique une focalisation sur le système du discours, c'est-à-dire sur les mécanismes de production de « ce qui peut et doit être dit » (Haroche et al., 1971, p. 23). Cet article est le premier à rendre compte de cette recherche et j'entends poser des jalons théoriques et méthodologiques pour conceptualiser mon objet de recherche. Loin d'être considéré comme une exigence morale, « Ne pas parler à la place des premiers concernés" est envisagé comme une pratique langagière collective et normative, plus exactement un registre (Agha, 2007) qui se manifeste à travers des pratiques de différents ordres et imprègne les interactions, les mises en discours, et les actes. L'objectif de cet article ${ }^{3}$ est précisément de 
Veniard, Marie. « 'Ne pas parler à la place des premiers concernés'. Étude d'une pratique politique d'éthique langagière chez des militants politiques pour les droits des étrangers en France. » Nouvelle

montrer qu'il s'agit d'une norme interactionnelle pervasive qui est récurrente à différents endroits du texte et se caractérise par sa multi-modalité. J'illustrerai ses manifestations aux niveaux mimo-postural, intradiscursif et interactionnel.

\section{1. « La voix » en politique : entre émancipation et instrumentalisation}

La question de la " voix» sera abordée telle qu'elle se présente pour certains acteurs, militants et intellectuels engagés, c'est-à-dire du point de vue de l'émancipation politique. La nécessité que les personnes concernées par les injustices soient celles qui s'expriment, qui décident de leurs revendications et de la manière dont elles organisent leur lutte est un thème qui traverse divers mouvements des luttes sociales. Michel Foucault en fait le point central de son Groupe d'Information sur les Prisons ${ }^{4}$ : " les masses n'ont pas besoin [des intellectuels] pour savoir ; elles savent parfaitement, clairement, beaucoup mieux qu'eux; et elles le disent fort bien. Mais il existe un système de pouvoir qui barre, interdit, invalide ce discours et ce savoir » (1972, p. 1176). Dans le champ des mobilisations pour le droit des étrangers, la pratique méta-pragmatique de « Ne pas parler à la place ... » relève d'une stratégie des militants soutiens pour marquer leur solidarité avec les personnes sans-papiers, afin que ces derniers disposent de l'espace pour s'approprier ou s'approprient une parole politique et la fassent entendre.

Ainsi, « Ne pas parler à la place ... » est une réponse à un problème qui caractérise toutes les luttes des " minorités » ou groupes dominés en quête de reconnaissance quand ils se mobilisent avec d'autres, et, plus largement, les situations de délégation de la parole. Pierre Bourdieu rappelle que toutes ces situations comportent le risque d'une confiscation de la parole, et du collectif, par le ou la porte-parole: " les individus à l'état isolé, silencieux, sans parole, n'ayant ni la capacité ni le pouvoir de se faire écouter, de se faire entendre, sont placés devant l'alternative de se taire ou d'être parlés » (par le porte-parole) (Bourdieu, 1984, p. 50), mais il conclut cependant qu'il « faut toujours risquer l'aliénation politique pour échapper à l'aliénation politique » (Bourdieu, 1984, p.49). C'est le pari que font, à notre sens, les militants qui mettent en pratique NP3.

La mobilisation étant menée conjointement par des militants sans-papiers et des militants par conscience, la question, sous-jacente, de l'autonomie des sans-papiers dans leur lutte est centrale dans les travaux de sociologique, et les travaux ethnographiques soulignent la nécessité scientifique d'adopter leur point de vue sur les mobilisations (Veron, 2019). Dans ce travail, nous nous focaliserons plus sur les soutiens et leurs pratiques langagières pour répondre à cette question de l'autonomie, qui passe notamment par l'appropriation de la parole.

A ce stade de la réflexion, il me semble important, à des fins surtout heuristiques, de distinguer entre le principe de " Ne pas parler à la place » et la, ou plutôt les, pratiques : ces deux faces de la question se construisent mutuellement, le principe cadrant la pratique et la pratique nourrissant le principe, pour le dire très vite. Cependant, du point de vue de la recherche, c'est-à-dire du point de vue adopté par le chercheur sur l'objet, travailler sur l'un ou l'autre est assez différent. Les passages que nous venons de citer, de Foucault ou Bourdieu, portent sur le principe de ne pas prendre la parole à la place des concernés. Ce principe moral est légitime dans le cas des migrants, qui sont « réduits au silence dans les espaces majoritaires, dans un régime de visibilité qui décide qui peut parler et qui peut être entendu » (Abbou, 2018, p. 87). L'invisibilisation et la stigmatisation sont confirmées par des études de corpus (Krzyżanowski et al., 2018 ; Bittencourt, 2021). Sur la question de la parole très précisément, Bittencourt (2021) souligne que, dans les médias généralistes, les personnes les plus citées sont le plus souvent des politiciens et des représentants des ONG. Les migrants (réfugiés et demandeurs d'asile dans cet article), " ne sont cités que dans $15 \%$ des textes. Plus de la moitié de ces textes ne font d'ailleurs que reprendre les paroles des immigrés publiées préalablement par d'autres journaux internationaux ou agences de presse » (Bittencourt, 2021, paragr. 39).

Le principe de « Ne pas parler à la place » trouve sa justification dans des constats de la réalité sociale, constats face auxquels les acteurs sociaux apportent différentes réponses. Je vais décrire celle proposée par des militants anti-racistes, mais d'autres existent, celle de militants queers par exemple (Abbou, 2018), qui manifestent « en tant que queers » (et non « au nom des migrants » par exemple) par solidarité avec les étrangers : "En parlant en tant que queers, ces collectifs diminuent le risque de dépersonnalisation et d'usurpation politique. En évitant le dispositif représentationnel - « parler pour » — ils évitent également la création d'un " nous " artificiel, et font plutôt le geste d'ajouter leur voix à celle des migrantes et des migrants qu'ils soutiennent, produisant une accumulation de voix » (Abbou, 2018, p. 91). Avant de décrire plus en détail la pratique de « Ne pas parler à la place », il convient de la situer, par rapport à une autre 
Veniard, Marie. « 'Ne pas parler à la place des premiers concernés'. Étude d'une pratique politique d'éthique langagière chez des militants politiques pour les droits des étrangers en France. » Nouvelle

avec laquelle ceux qui la pratiquent l'opposent (l'appropriation discursive) et par rapport à son milieu d'émergence, le champ militant.

\section{1. Une norme construite en opposition à l'appropriation discursive}

La norme interactionnelle « Ne pas parler à la place des premiers concernés » (désormais «NP3 ») présente, dans sa formulation, une négation polémique (Bres, 1999) : elle s'oppose, pour les militants des collectifs concernés, à une norme dominante que l'on peut verbaliser par un énoncé qui serait « parler à la place des premiers concernés ". L'exemple (1), tiré de l'enquête rapidement évoquée en introduction (voir la section 2 pour les détails), verbalise explicitement ces injonctions. Ce message est publié après le décès d'un jeune exilé dans un squat: le compte-rendu qu'un journal local a fait de la mort de ce jeune homme est critiqué dans ce post qui figure sur la page d'un collectif de sans-papiers ${ }^{5}$.

(1). II semble important de rappeler encore une fois qu'il est primordial que la parole des personnes concernées soit mise en avant, les personnes en «soutien » ne parle pas mieux de la situation que celleux qui la vive. Merci donc aux journalistes de faire l'effort de venir parler aux premier.e.s concerné.e.s et aux soutiens de ne pas se mettre en avant. Surtout dans une situation comme celle-ci (Gilets Noirs de [ville moyenne], 10 octobre 2019, post Facebook de la page d'un collectif de sans-papiers, orthographe d'origine).

On constate ici une rare mention explicite de l'impératif où " ne pas se mettre en avant " (variante de NP3) se concilie avec «faire émerger la parole des premiers concernés » (cf « faire l'effort de venir parler aux premier.e.s concerné.e.s »), les deux énoncés étant dans une relation de réciprocité. Le journal se serait rendu coupable de ce qu'on appelle dans le langage courant l'appropriation discursive.

Dans une approche critique, Paveau la définit comme "les manières qu'ont certain.e.s locuteurices de parler à la place des autres" (2017, p. 143). Elle défend l'idée selon laquelle " [p]arler à la place de celleux qui sont opprimé.e.s, c'est accomplir un triple geste : s'approprier leur point de vue subjectif, les destituer de leur possibilité de s'interpréter elleux-mêmes et produire des signes de domination voire d'oppression » (Paveau, 2017, p. 154). Le point de vue de Paveau est ici descriptif mais passe à une dimension prescriptive quand il est question de renverser cette appropriation discursive par des « dominants », ce qui n'est pas simple : "L'action politique impliquée par cette réflexion n'est pas en effet d'amener les subalternes à la parole (ce qui constituerait un point de vue "charitable », variante de l'appropriation discursive) mais d'engager une lutte contre la subalternité elle-même » (2017, p. 142). Pour Paveau (2017), " amener les subalternes à la parole » constitue une manière dissimulée de rejouer la relation de domination, une façon d'esquiver le problème.

Canut (2021) cite par exemple le cas de productions artistiques animées des meilleures intentions du monde, celles qui se réclament de la " voix des migrants », c'est-à-dire de l'idée de " donner la parole " à un groupe opprimé - et non de « ne pas parler à sa place $»^{6}$. Bien que nées d'intentions généreuses, ces productions révèlent, pour elle, un régime linguistique idéologique qui porte les traces des passés coloniaux. Malgré tout, des travaux en sciences politiques montrent que des contextes de politique locale ou plus explicitement d'éducation politique similaires à ceux de notre enquête, c'est-à-dire qui impliquent la présence d'intermédiaires, peuvent permettre l'émergence d'une subjectivation politique (Talpin, 2016b ; Tarragoni, 2014).

Les réflexions politiques de Canut (2021) ou Paveau (2017) peuvent avoir pour conséquence d'évaluer la qualité de la manière dont un agent tente de réaliser l'action de « ne pas parler à la place des premiers concernés ". C'est, à mon sens, la limite, en sciences du langage, d'une approche par le principe, et c'est pourquoi je me pencherai sur les pratiques. Ainsi, statuer sur l'efficacité de ces pratiques ne sera pas notre objectif, d'autant plus que cela demanderait d'être présent sur le terrain sur une très longue période. Je partirai de l'observation de terrain suivante: les militants pour les droits des étrangers que j'ai eu l'occasion d'observer, engagés au quotidien, m'ont semblé adopter de manière récurrente diverses pratiques pour favoriser, faire émerger, laisser de la place à la parole de militants sans-papiers, dans une relation d'alliance entre les deux collectifs (voir section 2). C'est de cette entreprise dont je souhaite rendre compte, de manière descriptive ${ }^{7}$. Afin de mener à bien ce projet, il est nécessaire de replacer NP3 dans sa sphère d'activité d'origine, le milieu militant. On peut alors envisager cette pratique par rapport à d'autres pratiques de réflexivité langagière intégrées à l'action politique.

\subsection{Activité militante et réflexivité langagière}


Veniard, Marie. « 'Ne pas parler à la place des premiers concernés'. Étude d'une pratique politique d'éthique langagière chez des militants politiques pour les droits des étrangers en France. » Nouvelle

De nombreux militants ont une conscience réflexive du pouvoir qui peut être exercé par la parole, comme l'ont montré Abbou (2018) chez les féministes libertaires, Husson (2017) au sein d'une communauté féministe en ligne et Him-Aquili (2018a) chez les anarchistes. Les militants du collectif de soutiens aux migrants sont familiers avec " une lecture du discours comme espace de pouvoir " (Abbou, 2017, paragr. 2). Ils produisent aussi, en entretien, ce que Abbou appelle des « métadiscours sur ces pratiques, qui visent précisément à les définir comme une critique pratique » (Abbou, 2017, paragr. 3) dans une tentative d'abolir la frontière entre théorie et pratique (ou discours et action). C'est cette tentative d'abolition que réalise la pratique de « Ne pas parler à la place».

Ces trois autrices montrent qu'on peut établir un lien entre des discours théoriques et idéologiques et des pratiques langagières concrètes, dans une articulation entre l'identité du groupe et ses positionnements et revendications : les écrits libertaires sur le pouvoir et l'écriture inclusive pour Abbou (2018); l'éthique du care et le trigger-warning pour Husson (2017) ; deux formations discursives en opposition concernant la régulation de la parole chez les anarchistes et l'ordre interactionnel chez Him-Aquili (2018b).

Un paramètre important pour comprendre ces méta-discours dans leur contexte d'apparition est de les replacer dans la sphère d'activité qui leur donne sens : le milieu militant. Abbou (2018) insiste ainsi sur la conception du discours comme un lieu de lutte (à l'instar d'autres lieux: l'espace public, l'espace médiatique ...) : «le discours est donc appréhendé, non seulement comme un lieu du politique, mais également comme une pratique politique » (paragr.28) et le discours partagé qui émerge « repose sur une appréhension de la langue comme lieu de lutte » (paragr. 17). La question de savoir « comment lutter par le langage? » se traduit parfois par des choix locaux, les désignations (Veniard, 2019, sur les désignations des personnes migrantes), mais cela ne représente qu'une partie du phénomène et d'une entreprise qui s'avère de toutes façons ardue (Krieg-Planque, 2018). Afin de montrer la manière dont NP3 irrigue les pratiques à différents niveaux de fonctionnement du langage, on donnera dans cet article une large place à l'analyse de données, dans une perspective volontairement empirique.

Dans le cadre de ce travail, on adoptera d'une part une position descriptive (vs prescriptive), et d'autre part une perspective émique (Olivier de Sardan, 1998), c'est-à-dire que je me placerai du point de vue des acteurs pour qui NP3 fonctionne comme une éthique. D'un point de vue analytique, je ne me prononce pas sur l'intérêt éthique de pratiquer, ou non, NP3, ni sur la qualité de sa réalisation ${ }^{8}$ par les locuteurs cités mais sur la manière dont la langue est productrice de ressources pour l'exprimer, ressources qui vont servir à actualiser un éthos militant anti-raciste.

\subsection{Une idéologie langagière : l'éthique langagière}

La pratique NP3 relève des idéologies langagières, c'est-à-dire selon Kroskrity (2009, p. 497), « [a] set of diverse beliefs, however implicit or explicit they may be, used by speakers of all types as models for constructing linguistic evaluations and engaging in communicative activity ». Les idéologies langagières postulent une articulation, via l'indexicalité, entre d'un côté les discours réflexifs sur les langues et leurs usages et l'expérience, les objets, le monde de l'autre

Je parlerai donc, pour les locuteurs rencontrés au cours de mon enquête, d'éthique langagière, en tant qu'il s'agit d'une conduite morale adoptée par un locuteur, conduite qui vise à actualiser au niveau langagier son engagement et ses valeurs. L'éthique langagière est ancrée dans une situation sociale et historique donnée. Elle repose sur le postulat, partagé par les linguistes aussi bien que par les nonlinguistes, que le langage constitue un pouvoir d'action sur le monde, que par le langage on actualise son rapport aux personnes et au monde, qu'une pratique langagière permet de rendre ce rapport visible par les autres, dans un monde construit et perçu via des dynamiques dialogiques. L'éthique langagière est recherchée, mise en œuvre et considérée telle par les militants-soutiens. Le chercheur ne préjuge pas de sa qualité.

\subsection{Le registre « Ne pas parler à la place »}

Les analyses de la section 3 mettront en avant la récurrence des marques de la norme « Ne pas parler à la place ». Je fais l'hypothèse que cette norme interactionnelle constitue un registre, au sens que lui donne Agha (2007) :

a linguistic repertoire that is associated, culture-internally, with particular social practices and with persons who engage in such practices 
Veniard, Marie. « 'Ne pas parler à la place des premiers concernés'. Étude d'une pratique politique d'éthique langagière chez des militants politiques pour les droits des étrangers en France. » Nouvelle

Revue Synergies Canada, № 15 (2022)

[...] « The utterance or use of a register's forms formulates a sketch of the social occasion of language use, indexing contextual features such as interlocutor's roles, relationships, and the type of social practice in which they are engaged (p. 24-25).

Agha (2007) souligne par ailleurs le fait que les indices qui participent du processus d'entextualisation (la mise en texte) cooccurrent, ce qui se révèlera cohérent avec la dimension multimodale et multidimensionnelle de NP3 (voir section 3.). On trouve chez Telep (2018) une mise en pratique très éclairante et très détaillée de cette notion, sur le registre de la " whitisation » (parler comme un Blanc, pour un Africain).

Considérer les manifestations langagières de NP3 comme un registre, un répertoire de formes et de pratiques interactionnelles permet une posture descriptive en laissant de côté, un temps, la normativité associée à NP3 en contexte militant, dont nous gardons toutefois une trace via la formulation au mode impératif (« ne pas parler »).

\section{Méthodologie : une enquête sur les luttes des personnes sans-papiers}

\section{1. Être sans-papiers}

Quand les personnes sans-papiers parlent de leur situation, elles disent « mon problème », ou « je n'ai pas les papiers »: des papiers, elles en ont, leur passeport généralement, mais ce n'est pas «le bon papier », il faut " gagner les papiers », c'est-à-dire obtenir un titre de séjour, qui sera d'abord temporaire (1 an), puis plus stable (statut de résident de 10 ans, ou nationalité). L'ethnologue Frédérique Fogel (2019) donne une description très précise du parcours éprouvant qu'est la quête du titre de séjour. Celles et ceux qui revendiquent l'appellation de « sans-papiers » ont en réalité beaucoup de papiers, toutes les preuves qu'ils et elles accumulent pour constituer leur dossier de demande de titre de séjour. II est important de dire que ces personnes alternent des phases de légalité de leur séjour à des phases d'illégalité. Fogel (2019) parle de « migrants immobilisés », pris dans une temporalité provisoire qui peut durer plus de 10 ans. Ainsi, j'ai vu une personne obtenir un titre provisoire après 22 ans de séjour.

\subsection{La lutte : les militants sans-papiers et les militants par conscience}

Puisque la cause du droit des étrangers engage à la fois des militants par conscience et des immigrants étrangers (Però et Solomos, 2010), la question de la voix des "subalternes » pour reprendre le mot de Spivak (2010) est essentielle et la plupart des militants soutiens en ont une conscience réflexive, qui irrigue leurs pratiques langagières. Par ailleurs, les personnes étrangères sans-papiers ont une maitrise variable du français qui est la langue de prise de parole publique (mais pas nécessairement la langue unique dans laquelle se déroulent les réunions). La situation de contact des personnes sans-papiers et des soutiens est celle d'une relative asymétrie de compétences (langagières, militantes en France ${ }^{9}$ ) et d'intérêts ne se recouvrant que partiellement : les uns cherchent en priorité une régularisation, les soutiens les accompagnent, tout en menant, par-là et pour certains, une lutte anti-raciste concrète qui peut prendre une valeur dans un autre cadre d'action (un parti politique par exemple). On le voit, la question se pose très différemment dans le cas de la lutte féministe, dans laquelle les militantes s'expriment souvent dans leur langue maternelle, sont pour certaines, du fait de leur vie professionnelle et de leur position d'universitaires ou d'artistes, habituées des prises de parole publiques. Enfin, le contexte de lutte politique rend la mise en pratique de " Ne pas parler à la place » différente de ce qu'elle peut être dans le cas d'un projet éditorial visant à rendre publiques les voix de celles et ceux que l'on n'écoute pas ou peu : les militants sans-papiers sont amenés à prendre la parole publiquement, à rencontrer et chercher à convaincre des interlocuteurs officiels, à négocier avec d'autres collectifs, etc.

\subsection{L'enquête}

Les données sont tirées d'une enquête de terrain d'un an et demi (février 2018-novembre 2019) auprès de deux collectifs de quartier, un collectif de soutien aux migrants (selon le terme choisi par ces acteurs) et un collectif de sans-papiers (selon, là encore, le terme des acteurs eux-mêmes).

Ces deux collectifs sont en relation d'alliance (Mathieu, 1999), partagent des causes communes (lutte contre racisme "d'État » et les violences policières) et organisent ensemble de nombreuses actions (manifestations, repas de soutien, évènements de mobilisation). Sans que cela fasse l'objet d'une revendication de la part des soutiens, les relations entre les deux collectifs présentent des similarités avec le « community organizing » (Talpin, 2016a), c'est-à-dire du processus permettant « aux gens de se 
Veniard, Marie. « 'Ne pas parler à la place des premiers concernés'. Étude d'une pratique politique d'éthique langagière chez des militants politiques pour les droits des étrangers en France. » Nouvelle

mobiliser par eux-mêmes pour obtenir des gains substantiels et bénéficiant aux groupes les plus défavorisés " (définition des acteurs du terrain citée dans Talpin, 2016a, p. 65). Talpin donne la définition suivante : le community organizing est « un projet d'émancipation des classes populaires qui requiert la constitution de contre-pouvoirs à l'influence de l'État et des forces du marché. II repose sur une participation en nombre de la population, ce qui suppose l'intervention de professionnels déployant des méthodes de mobilisation fortement rationalisées »(2016a, p. 66). En fonction des groupes, Talpin (2016a) note que la place de l'idéologie est plus ou moins forte. Dans les deux collectifs auprès desquels nous avons mené notre enquête, l'idéologie est très présente, du fait de leur vocation contestataire (voir la distinction posée par M. Pette (2019) entre associations attestataires et contestataires dans le champ de la lutte pour les droits des étrangers).

\section{La mise en pratiques du registre NP3}

Dans nos analyses, nous exploiterons en priorité la transcription de l'enregistrement d'une réunion du collectif de sans-papiers (12 mars 2019), à laquelle assistent les militants soutiens, comme c'est l'habitude ${ }^{10}$. Nous complèterons ces données par d'autres tirées de notre journal de terrain, et par des documents de communication numérique de différents collectifs (messages reçus sur des listes de diffusion, publications sur les réseaux sociaux). Nous mènerons une description des différents lieux d'inscription de la norme interactionnelle, aux niveaux kinésique, discursif et interactionnel. Dans les extraits, tous les noms ont été modifiés.

\subsection{Gestes}

La réalisation de NP3 peut avoir lieu par des gestes : on développera dans l'extrait suivant un regard et un déplacement du haut du corps. II s'agit de l'accompagnement d'un membre du collectif des sans-papiers à une permanence syndicale au sujet d'un conflit avec son employeur

Accompagnement Abdoul Dramane, qui maîtrise bien le français, à la permanence de l'union locale de la CGT (Confédération Générale du Travail), [ville], pour une question sur régularisation par le Cerfa. Je suis avec Imen, membre du collectif de soutiens, et soutien au collectif des sans-papiers de l'arrondissement comme moi.

Nous sommes reçus par une syndicaliste: nous sommes tous les trois face à elle, AD est le plus en face, nous sommes deux femmes (blanche / d'origine maghrébine) qui ont été identifiées comme des soutiens et un homme, noir, identifié comme sans-papier. Elle regarde avec insistance $^{11}$ Abdoul Dramane en lui posant des questions sur son lieu d'habitation, de travail. II ressort alors que cette section locale du syndicat ne peut pas traiter son dossier (il habite dans le 92 et la permanence est dans le 75). La jeune femme se tourne alors vers nous et évoque la procédure en général. Nous avons des questions. Delphine, la responsable de la permanence, vient nous aider, elle passe quelques coups de fil et identifie un interlocuteur pertinent dans le 92.

\section{(2). Journal de terrain, $4 / 02 / 2019$}

Avant de développer une interprétation de cette séquence, il faut préciser que les interprétations que je propose ici résultent de la compréhension que j'ai eu de ce moment, compréhension étayée par l'expérience de multiples interactions de ce type. Par ailleurs, ces explications prennent sens du fait de l'analyse d'autres interactions, convergentes, développées dans la suite de la section.

La syndicaliste qui nous accueille a catégorisé les participants sans recourir à des questions, tant les catégories complémentaires, racialisées, que sont les soutiens et les sans-papiers s'imposent comme grille de lecture. Dans un premier temps, elle pose directement, et par l'insistance de son regard, ostensiblement, exclusivement, ses questions sur ses lieux d'habitation et de travail à Abdoul Dramane. C'est ce regard que j'interprète comme la réalisation de NP3 par un geste : la syndicaliste signifie ainsi qu'elle cherche à ce qu'Abdoul Dramane présente sa situation lui-même, et donc parle pour lui-même. Elle s'assure qu'aucune des femmes soutiens ici présentes (elle-même et nous) ne parle à sa place. On peut imaginer que la syndicaliste accomplit NP3 pour elle-même mais également pour nous, pour défendre devant nous une image positive d'elle-même, dans les termes de la préservation des faces. Dans un second temps, s'adressant à nous, elle change de registre communicationnel pour expliquer la procédure dans son ensemble, la suite des actions à entreprendre, auxquelles elle suppose que nous prendrons part. Ce second temps suggère que la syndicaliste anticipe une asymétrie supposée des compétences (anticipation sur un niveau de langue supposé, connaissances en droit pour la régularisation). Cette 
Veniard, Marie. « 'Ne pas parler à la place des premiers concernés'. Étude d'une pratique politique d'éthique langagière chez des militants politiques pour les droits des étrangers en France. » Nouvelle

asymétrie s'avère pertinente dans certains cas, mais pas toujours. Dans le cas présent, Abdoul Dramane maitrise bien le français et connait mieux que nous deux, accompagnantes, le processus de régularisation par le travail. Ces deux mouvements sont en tension : l'asymétrie supposée des compétences prenant le pas sur NP3.

Bien sûr, on ne peut pas exclure que les règles de politesse influent également. Cette jeune femme a devant elle trois personnes. Elle peut difficilement, au nom de NP3, les ignorer tout au long de l'interaction. Cependant, la prise en compte de ces trois interlocuteurs n'est pas réalisée par un regard balayant, ce qui aurait été le cas si la situation avait été gérée uniquement selon les normes de politesse, mais elle est bien régulée par NP3, qui structure la distribution des regards.

\subsection{L'intradiscours : la mise en avant de l'agentivité des personnes sans-papiers}

Le registre NP3 s'actualise également au niveau de l'intra-discours ${ }^{12}$, c'est-à-dire, dans les termes de l'analyse de discours, le fil linéaire du discours, traversé par l'interdiscours, c'est-à-dire, pour résumer rapidement, de discours autres, d'idéologie. Nous décrirons la récurrence dans la mise en avant de l'agentivité des personnes sans-papiers. C'est en particulier très visible sur des textes destinés à circuler publiquement (communiqués, messages de type compte-rendus ou messages sur des listes de diffusion). Ces textes circulent sur des listes ou des réseaux sociaux à destination principalement des militants par conscience. La mention de prises de parole réalisées par les sans-papiers, l'organisation de leur visibilité et la demande de retrait des soutiens sont régulières. On peut lire par exemple sur un appel à manifestation: "Les prises de parole seront prioritairement celles des exilé.e.s. [...] Les soutiens des partis politiques et des syndicats sont les bienvenus, mais leurs visuels de devront pas cacher ceux des exilés et de leurs soutiens associatifs, citoyens, bénévoles et militants » (collectif $X$, Calais, manifestation du 8 mai 2019, post facebook). L'exemple (3) illustre un cas où la règle de la visibilité par les militants sans-papiers n'est pas tout à fait respectée.

Modalités de cortège

Les cortèges de tous les collectifs de sans-papiers et migrantEs seront en tête de manifestation suivis par les syndicats puis ceux des associations et enfin des organisations politiques.

\section{La présence des cortèges de sans-papiers et migrantEs en tête de manifestation a un contenu politique clair que nous demandons donc à toutes et à tous de respecter.}

Pour la même raison il n'y aura pas de « carré de tête " qui précèderait les sans-papiers et migrantEs. Mais à la demande des collectifs de sans-papiers qui veulent mettre en avant la nécessité d'unité et d'égalité la banderole de tête qui ouvrira les cortèges des collectifs sera tenue, aux côtés des sanspapiers, par une représentation de touTes les signataires.

(3). Manifestation du 18/12/2018, Journée internationale des migrants, « Égaux, égales, personne n'est illégal », Mail du 12/12/2018 (les soulignements en gras figurent dans le document d'origine)

Cet extrait de programmation de manifestation souligne un dispositif stratégique de mise en avant des personnes sans-papiers, physiquement, en tête de manifestation et lors de la prise de parole de fin de manifestation, position de visibilité qui marque des positions de pouvoir, dans la manifestation, la tête de cortège et après, lors des prises de parole.

La première personne du pluriel indique que l'énonciateur est un soutien qui s'adresse à des soutiens en parlant des SP. On retrouve donc la même situation que plus haut, à savoir que la réalisation de NP3 se fait pour les personnes sans-papiers, mais fait sens aussi pour les soutiens.

La formulation de l'action, adressée aux associations, syndicats et partis politiques, qui vise à matérialiser NP3 est très explicite quant au positionnement du cortège de sans-papiers et à son sens politique. Dans ce cadre, la proposition que des représentants des organisations signataires défilent en tête au côté des collectifs des sans-papiers constitue une contradiction et contrevient au respect de NP3. Cette contradiction est résolue, à mon sens, par la mise en avant de l'agentivité forte des collectifs de sans- 
Veniard, Marie. « 'Ne pas parler à la place des premiers concernés'. Étude d'une pratique politique d'éthique langagière chez des militants politiques pour les droits des étrangers en France. » Nouvelle

papiers dans cette décision (« à la demande ») : les organisations ne se mettent pas en avant, elles acceptent une proposition des militants sans-papiers. Mettre en pratique « Ne pas parler à la place », c'est ici justifier d'une décision, et anticiper des critiques éventuelles.

\subsection{En interaction : incitation à l'action et interruptions.}

Le dernier niveau d'analyse sera le niveau pragmatique, à partir de données interactionnelles. Deux extraits seront commentés, l'un montrant la réalisation d'une incitation à l'action (acte de parole que l'on place entre la suggestion et l'ordre) et l'autre une interruption et sa réparation. Avant de passer à l'analyse, il convient de donner quelques caractéristiques des interactions militantes, ce qui permettra de mieux saisir leur spécificité. Les réunions du collectif de sans-papiers, relativement formelles, présentent certains traits des assemblées générales anarchistes décrites par Him-Aquili (2018a) : les anarchistes ont une forte conscience réflexive du pouvoir que représente la parole et la prise de parole et se sont dotés d'un ensemble de règles qui visent à encadrer ce pouvoir et garantir des relations horizontales entre les membres. Ainsi, la parole n'est pas libre, mais régulée par un preneur de tour qui prend note des participants qui souhaitent s'exprimer et par un animateur qui veille au respect de l'ordre du jour. Les prises de parole peuvent être limitées dans le temps (à quelques minutes). On aboutit alors à une interaction dans laquelle des interventions successives ne se répondent pas nécessairement directement l'une à l'autre (la structure en paires adjacentes, de type question-réponse n'est pas la structure de base). Ce point jouera un rôle dans la description de l'interruption (voir plus bas). Par ailleurs, et à côté de conflits ouverts, les participants se livrent - comme dans d'autres lieux - à un important travail de figuration et de protection des faces.

Dans les réunion du collectif de sans-papiers, l'incitation à l'action se base, comme chez les militants anarchistes, sur l'auto-désignation (Him Aquili, 2018a) : l'action est présentée, les tâches sont identifiées par le locuteur et les présents attendent que des membres du collectif se proposent pour une tâche, à l'oral ou via un tableau de répartition des tâches. Il est impensable de donner un ordre directement quand des relations horizontales régissent les rapports entre les membres d'un collectif.

(4). Laura [à propos de la mobilisation pour une manifestation] : alors / pourquoi je dis tout ça / eh bien parce que je me dis bon ben / il nous reste quelques jours pour arriver à faire en sorte d'avoir ce beau cortège / donc la question qui se pose c'est qu'est-ce qu'on peut faire pour avoir un beau cortège d'ici samedi alors Ousmane il a dit qu'il y avait des autocollants donc si chacun en récupère il peut autour de soi au foyer dans la rue après des voisins / c'est pareil y a des tracts on peut en prendre qqs uns et les distribuER à : ses amis, ses voisins ses collègues à ses cousins etcetera / p'tête que ça vaut le coup d'appeler les gens du collectif qui sont pas là ce soir / que vous connaissez leur dire qu'c'est très important de venir samedi si y travaillent pas /à la manifestation et de donner le rendez-vous à midi à ménilmontant $\mathrm{P}^{\prime} T E T$ e que : VOIlà : si vous êtes plusieurs sur un foyer c'est possible d'organiser du porte à porte Réunion du collectif des militants sans-papiers, 12/03/2019, enregistrement.

(5). Stéphanie : [à propos du recensement de lieux de travail en prévision d'une série de grèves] l'idée après c'est de dire ah sur cette entreprise là on voit qu'il y a monsieur machin, monsieur monsieur et on a 15 personnes pour euh 80 personnes qui travaillent ça peut être le cas pour une boite d'interim / après il faudrait le faire Fatoumata l'a dit Ousmane l'a dit aussi pour le 20 / donc le 20 c'est mercredi prochain / donc euh il faut discuter entre nous comment on va pouvoir le FAlre p'te que bon moi là j'ai une idée qui me vient / on peut le faire mardi prochain, on a ce temps-là déjà et on peut peut-être aussi euh / je sais pas si ça pose un problème d'envoyer ce type d'information sur WA mais : ça serait une possibilité / $(3 \mathrm{sec})$ parce qu'il y a la manif samedi donc euh Réunion du collectif des militants sans-papiers, 12/03/2019, enregistrement.

(4). Transcription d'un extrait de réunion du collectif des militants sans-papiers (12/03/2019) 
Veniard, Marie. « 'Ne pas parler à la place des premiers concernés'. Étude d'une pratique politique d'éthique langagière chez des militants politiques pour les droits des étrangers en France. » Nouvelle

Dans le contexte anarchiste comme dans le contexte des militants pour les droits des étrangers, tout acte relevant de l'injonctif est donc considéré comme un Face Threatening Act (désormais FTA) très sérieux, ce qui explique ici les adoucisseurs nombreux utilisés par la militante soutien dans l'exemple (4) pour inciter les militants sans-papiers à l'action (participer à la mobilisation pour la manifestation, en distribuant des tracts, etc.) : déréalisation (système hypothétique "si chacun peut ", modalité de possibilité "il peut », « on peut »; pronom incluant tous les participants à la réunion « on » à valeur de " nous " ${ }^{13}$ (les soutiens et les sans-papiers dans « on peut faire pour avoir un beau cortège »). L'analyse de l'exemple (5) est similaire.

Cet énoncé et la surabondance de marques viennent illustrer la tension à l'œuvre lors de la réalisation de NP3 : comment maintenir NP3 quand on est dans la situation de suggérer des modes d'action à des personnes qui ne sont pas membres du même collectif? Bien sûr, on pourra répondre à cette question qu'il suffit de ne pas de trouver dans cette situation, mais cela ne rend pas compte des pratiques des acteurs. Ici, nous interprétons la surabondance du marquage de la modalité visant à circonscrire la portée de l'ordre comme une tentative de résolution de cette tension.

Dans le cadre des interactions militantes décrit plus haut, les interruptions n'ont pas lieu d'être puisqu'elles seraient le signe du non-respect de normes constitutives de l'habitus du groupe. Quand elles surviennent, dans les assemblées anarchistes, elles suscitent des excuses marquées que Him-Aquili (2018b) interprète non comme des marques de politesse (une réparation) mais comme un marquage de sa loyauté vis-à-vis des règles d'accès à la parole au sein du groupe. Ainsi, « [c]ouper la parole, et dans une moindre mesure parler en même temps que quelqu'un d'autre, semble constituer un comportement interactionnel fortement dévalorisé car indexé à une attitude autoritaire dont personne ne veut faire preuve ni être accusé et que tout le monde cherche à éviter publiquement" (Him-Aquili, 2018b, p. 10). Les interruptions et leur négociation interactionnelle sont donc régulées par l'idéologie langagière anarchiste, et, sans doute, plus largement, des groupes anti-autoritaires.

Dans le contexte de mon enquête, l'interruption constitue un FTA à double titre : en raison des normes de prise de parole dans le milieu militant mais également en raison de NP3, norme locale de la lutte pour les droits des étrangers. L'extrait suivant s'ouvre sur une prise de parole de Samba qui défend l'adoption de règles plus strictes pour le collectif. Julien anime la réunion et Alioune attribue la parole aux participants qui l'ont demandée. 
Veniard, Marie. « 'Ne pas parler à la place des premiers concernés'. Étude d'une pratique politique d'éthique langagière chez des militants politiques pour les droits des étrangers en France. » Nouvelle

Julien-S : on essaie de s'écouter parce qu'après [on a encore un sujet important à à traiter

2 Alioune-SP :

[on s'éCOUTE !]

3 Alioune-SP : on s'éCOUTE ! / bon Samba !

4 [léger brouhaha] Samba-SP : bonsoir tout le monde / ben c'est juste de rajouter ce que Stéphanie 5 vient de dire / que Ousmane / les autres / comme le vendredi dernier j'étais là avec: / parmi les 6 personnes / on était là vendredi $\downarrow$ on s'est discuté / il faut que on met en place / si on n'est pas met 7 une place je crois que on n'arrivera jamais / on n'arrivera jamais / comme Sté / Stéphanie vient de 8 dire quand on a: on a commencé à désin- à désinscrire les gens dans le whatsapp / ou à radier les 9 gens ou à: dans le whatsapp dans le groupe/ peut-être ça a démoralisé certains gens / pour moi 10 personnellement je préfère $\downarrow /$ même quand on est 10 personnes $\downarrow$ ou 20 personnes qui sont 11 SErieux / c'est mieux que avoir 100 personnes qui sont pas sérieux $\downarrow /$ moi je veux que on: on met 12 UNE règle / c'est ça que je veux / il faut que je: on met une règle ce soir $\downarrow$ / avant que on finisse / 13 voilà $\downarrow c^{\prime}$ est ça que je veux / $c^{\prime}$ est ça que je que je voulais dire

\section{$14 \quad(2 \mathrm{sec})$}

15 Julien-S : est-ce que y a besoin de: de j'sais pas de euh [c'est bon $\uparrow$

17 Julien-S : ah pardon excuse-moi, je pensais que t'avais fini / non mais vas-y continue hein si:

Alioune-SP $:$ il a fini=

Samba-SP : =ouais c'est bon

21 Julien-S est qu'y a besoin de traduction ?

22 X-SP : NON $\uparrow$

23 Samba-SP NON euh ça:

$24 \mathrm{X}$-SP : non si c'est le pulaar. (Echanges en pulaar et soninké en arrière-plan)

25 Alioune-SP : ouais (il valide une prise de parole)

(5). Transcription d'un extrait de réunion du collectif des militants sans-papiers (12/03/2019)

À la fin de la prise de parole de Samba, le volume de sa voix baisse et une pause longue s'ensuit (I. 1314 ), indice que Julien interprète comme la fin du tour de parole. II enchaine sur la suite de la prise de parole en proposant une traduction (I. 15), que Samba ne juge pas nécessaire (I. 16), mais Julien interprète cette intervention de Samba comme la continuité de son tour précédent (I. 17). II s'excuse pour ce qu'il réinterprète comme une l'interruption, ce qui déclenche une séquence de réparation qui constitue l'objet de notre analyse (I. 17-23): Alioune, puis Laura et enfin Samba confirment dans des énoncés enchainés l'absence d'interruption, et par là, l'absence d'offense.

Pourquoi interpréter cet échange comme une manifestation de NP3 plutôt que comme un échange de type « interruption - réparation - validation de la réparation »? Autrement dit, pourquoi cette possible interruption (c'est l'interprétation que fait Julien de l'interaction) suscite-t-elle un dérèglement de l'interaction pendant 5 tours de parole?

Premier point, cet échange est bien un échange de réparation après un possible FTA (l'éventuelle interruption), mais cette explication n'explique pas l'intégralité du passage : Alioune est preneur de tour, il lui revient de veiller à l'alternance des locuteurs, Samba est directement concerné par la réparation. Cependant, on note une autre intervention, celle, enchainée, de Laura qui confirme elle aussi la réparation. Laura est une militante très expérimentée, pilier des deux collectifs : co-fondatrice du collectif de soutien et ayant accompagné la création du collectif de sans-papiers. Mon argument s'appuie sur cette diversité des statuts : pourquoi Laura interfère-t-elle dans l'échange? quel est le sens de son intervention? 
Veniard, Marie. « 'Ne pas parler à la place des premiers concernés'. Étude d'une pratique politique d'éthique langagière chez des militants politiques pour les droits des étrangers en France. » Nouvelle

plus précisément, pourquoi confirmer qu'il n'y a pas offense? Je propose l'hypothèse selon laquelle c'est la norme interactionnelle NP3 qui fonde l'intervention de Laura dans la mesure où l'injonction à ne pas interrompre les premiers concernés quand ils parlent a pu être non respectée, ce qui se marque par la confusion autour du fait que Samba ait ou non été interrompu. Laura viendrait là confirmer, non pas auprès de Samba, mais de Julien qu'il n'a pas commis d'infraction à NP3, et peut-être plus largement à l'assemblée - voire à un sur-destinataire - qui ne souhaite pas voir ou laisser passer dans leurs réunions ce qui serait un acte contraire à NP3. Cette séquence est à replacer dans la dynamique de l'interaction : l'intervention de Laura, du fait du poids de la locutrice dans le groupe, permet de sortir de la confusion, confirmant l'absence d'offense et incitant ainsi à la poursuite de la réunion, ce que laisse suggérer sa voix calme. Alioune reprend la main sur les prises de parole un peu plus tard (I.25).

\section{Conclusion}

Au fil de cet article, nous avons montré que la pratique langagière de « ne pas parler à la place des premiers concernés " fonctionne de manière multi-modale (langage, gestes), multi-dimensionnelle (lexique, syntaxe, ordre interactionnel), dans des contextes différents (permanence syndicale, réunion de collectif, etc.), sur des supports différents (écrit/oral). II s'agit bien d'un registre qui se répartit sur des marqueurs de nature et de niveau différents. Au vu de cette régularité dans l'usage, on peut alors conclure à la dimension systématique de ce registre qui dépasse les pratiques et les intentions morales d'un individu. La dimension injonctive transparait dans les extraits (cf extrait 1,3) qui soulignent le caractère impératif du respect de la norme interactionnelle. L'éthique langagière ainsi est constituée par les pratiques et discours politiques du champ.

À l'heure actuelle nous constatons un regain de force du militantisme (anti-raciste, féministe, identitaire, religieux, conservateur...), "ne pas parler à la place ..." circule dans certains de ces milieux et fonctionne comme un impératif doté d'une dimension morale et injonctive forte, allant contre les pratiques habituelles de nombreuses sphères de la société. II me semble important de l'étudier " dans un de ses milieux naturels » de déploiement et de le replacer par rapport aux pratiques militantes, qui, seules, lui donnent sens, ou, du moins, qui lui donnent son sens premier. C'est la manière dont je vois ma contribution de chercheuse à la société, comprendre comment fonctionne « Ne pas parler à la place ...» et ce que cela veut dire plutôt que de le louer ou le condamner. Cette position permet notamment de décrire les tensions et ambivalences dont s'accompagne l'actualisation de NP3 par les militants soutiens, aspect qui constitue la suite du présent travail (voir Veniard à paraitre en 2022). L'analyse des conflits permet de mettre en évidence encore plus clairement la dimension normative de cette pratique langagière, dont j'ai tenté de montrer, ici, la systématicité et la multi-modalité.

\section{Notes}

${ }^{1}$ Les personnes associées à une mobilisation mais n'étant pas directement concernées sont parfois appelées des "alliés » ou des "complices », chaque terme étant discuté dans l'espace militant des mobilisations concernées. II s'agit là de réflexions méta-discursives interne aux groupes en question et non de positionnements académiques.

2 La notion de «travail de figuration » (face work), telle que définie par Goffman comme «tout ce qu'entreprend une personne pour que ses actions ne fassent perdre la face à personne (y compris à ellemême) " (1974, p. 15). Pour effectuer ce travail de figuration, il faut tenter d'atténuer ou d'éviter de produire des Face Threatening Acts (Actes menaçants pour la face, FTA) et produire des Face Flattering Acts (Actes Flattants pour la face, FFA) envers autrui. L'ensemble des moyens utilisés pour atténuer un FTA sont appelés softeners (intonation, mimiques, modalisateurs, formulations indirectes, minimisateurs ...).

${ }^{3}$ La dimension idéologique de « Ne pas parler à la place ... » est traitée de manière approfondie dans Veniard (à paraitre en 2022), sous l'angle de l'analyse de discours.

${ }^{4}$ Bagault (2017, p. 23) précise d'ailleurs le caractère éphémère du GIP : « Ayant pour objectif de donner la parole aux détenus puis de leur passer le relais, le Gip s'autodissout en décembre 1972 au profit du Comité d'action des prisonniers (Cap), emmené par l'écrivain militant, ex-détenu, Serge Livrozet ». Le dispositif visant à permettre l'émergence de la parole des prisonniers a disparu, ayant rempli sa fonction. 
Veniard, Marie. « 'Ne pas parler à la place des premiers concernés'. Étude d'une pratique politique d'éthique langagière chez des militants politiques pour les droits des étrangers en France. » Nouvelle

\begin{abstract}
${ }^{5}$ Nous ne connaissons pas les circonstances de production de cette page d'un compte de réseau social qui, si elle s'affiche comme étant celle d'un collectif de sans-papiers, est vraisemblablement animée par des militants soutiens. Cette répartition des tâches n'est pas inhabituelle dans ce milieu.
\end{abstract}

${ }^{6}$ À supposer qu'on puisse nettement distinguer, dans les faits, ces deux principes, ce qui serait à évaluer.

7 Descriptif et prescriptif ne s'excluent pas, mais, autant que possible, ne doivent pas être mêlés. La restitution de l'enquête auprès des collectifs a été repoussée à 2021, mais certains militants l'attendent de sorte à générer un débat sur la question de la parole au sein des collectifs et les pratiques à privilégier. Autrement dit, mes observations descriptives seront entendues avec une oreille prescriptive. Je pourrais moi-même être amenée à donner mon avis sur certains points.

${ }^{8}$ De sorte à répondre à la question : « est-ce qu'en disant cela ce locuteur réalise-t-il bien « ne pas parler à la place des premiers concernés »? ou réalise-t-il plutôt " parler pour », "donner voix à »? selon les oppositions politiques mentionnées plus haut.

${ }^{9}$ On réfère ici à un manque de connaissance des répertoires d'action et des acteurs de la mobilisation sociale en France, et non à un manque de compétence militante dans l'absolu. Parmi les personnes sanspapiers avec je me suis entretenue, plusieurs ont mentionné avoir participé, dans leur pays d'origine, à des grèves du travail, à des mouvements sociaux, ont manifesté un esprit contestataire et ces investissements ont parfois été réprimés. Certains membres du collectif ont l'expérience de conflits du travail en France.

${ }^{10}$ Réciproquement, des militants sans-papiers assistent aussi régulièrement aux réunions du collectif de soutiens.

11 Je souligne, dans mon journal de terrain.

12 On fait référence ici à l'opposition entre intradiscours (la linéarité de la parole) et interdiscours (ce qui renvoie au dialogisme, à la circulation des discours), courante en analyse de discours (voir Maldidier, 1990).

${ }^{13}$ Mais aussi parfois à valeur de « vous » (« il y a des tracts on peut en prendre quelques-uns »).

\title{
Normes de transcription
}

prénom + SP = militant sans-papiers

prénom $+\mathrm{S}=$ militant soutien

Tous les prénoms ont été remplacés.

$=$ enchainement rapide

/ pause courte

(2 sec) pause longue

$\downarrow \uparrow$ intonation descendante / montante

ça que je que voix plus basse

[ chevauchement

- mot coupé

( éclaircissements sur le sens, précision sur le contexte 
Veniard, Marie. « 'Ne pas parler à la place des premiers concernés'. Étude d'une pratique politique d'éthique langagière chez des militants politiques pour les droits des étrangers en France. » Nouvelle Revue Synergies Canada, № 15 (2022).

\section{Bibliographie}

Abbou, J. (2017). Cultures politiques du discours : Féminisme, anarchisme et rhétorique. Argumentation et Analyse du Discours, 18. https://doi.org/10.4000/aad.2329. (Consulté le 5 mai 2020).

Abbou, J. (2018). «Queer Solidarity Smashes Borders ». Une politique de la solidarité avec les mi-grants en tant que queers. Migrations Société, 173 (3), 79-97.

Agha, A. (2017). Registers of language. Dans In A. Duranti (éd.), A Companion to Linguistic Anthropology (p. 23-45). John Wiley \& Sons, Ltd.

Bagault, C. (2017). L'expérience du Gip ». Dans H. Lhérété (éd.), Michel Foucault : L'homme et l'oeuvre. Héritage et bilan critique (p. 22-23). Éditions Sciences Humaines.

Bourdieu, P. (1984). La délégation et le fétichisme politique. Actes de la Recherche en Sciences Sociales, 52(1), 49-55.

Bourdieu, P. (éd.). (1993). La Misère du monde. Seuil.

Bres, J. (1999). Vous les entendez? Analyse du discours et dialogisme. Modèles linguistiques, 20(2), 71-86.

Bittencourt, A. (2021). Les médias «grand public » et les médias "alternatifs » face à la "crise des migrants ». $\quad$ Communication len ligne], 38(1). https://journals.openedition.org/communication/13490\#tocto3n1 (Consulté le19 août 2021).

Canut, C. (2021). Can « migrants » speak? Voices, narratives and performances. Dans C. E. Skalle et A. M. Gjesdal, Transnational Narratives of Migration and Exile Perspectives from the Humanities (18-44). [En ligne].

Cissé, M. (1999). Parole de sans-papiers. La Dispute.

Fogel, F. (2019). Parenté sans papiers. Éditions Dépaysage.

Foucault, M. (1972). "Les intellectuels et le pouvoir" (entretien avec Gilles Deleuze, 4 mars 1972), L'Arc, 49, pp. 3-10, repris dans Dits et écrits, tome I, pp. 1174-1183.

Goffman, E. (1974). Les rites d'interaction. Les éditions de Minuit.

Greco, L. (2018). Dans les coulisses du genre : la fabrique de soi chez les Drag Kings. Lambert Lucas.

Haroche, C. ; Henry, P. et Pêcheux, M. (1971). La sémantique et la coupure saussurienne: Langue, langage, discours. Langages, 24, 93-106.

Him-Aquili, M. (2018a). Prendre la parole sans prendre le pouvoir. Réflexivité, discours et interactions dans les assemblées générales anarchistes et/ou autonomes. [Thèse de doctorat en sciences du langage, Université Paris Descartes].

Him-Aquilli, M. (2018b). Distribuer le pouvoir comme on distribue la parole : le rituel des «tours de parole» dans des assemblées générales anarchistes/autonomes. Semen. Revue de sémio-linguistique des textes et discours, 43, 18p.

Honneth, A. (2018). La lutte pour la reconnaissance. Folio Essais. (1ère édition 2013).

Husson, A. -C. (2017). Éthique langagière féministe et travail du care dans le discours. La pratique du trigger warning. Langage et société, 159, 41-61. 
Veniard, Marie. « 'Ne pas parler à la place des premiers concernés'. Étude d'une pratique politique d'éthique langagière chez des militants politiques pour les droits des étrangers en France. » Nouvelle

Krieg-Planque, A. (2018). " Lutter au sujet du langage fait partie du combat idéologique » - Entretien avec Alice Krieg-Planque. Agir par la culture, 53. https://www.agirparlaculture.be/alice-krieg-planquelutter-au-sujet-du-langage-fait-partie-du-combat-ideologiquel.

Kroskrity, P. V. (2009). Language Ideologies. Dans A. Duranti, A Companion to Linguistic Anthropology (496-517). Oxford, Blackwell Publishing.

Krzyżanowski, M. ; Triandafyllidou, A. et Wodak, R. (2018). The mediatization and politicization of refugee crisis in Europe [numéro thématique]. Journal of Immigrant and Refugee Studies, vol. XVI, no 12.

Lepinay (de), A. (2019). Organisons-nous! Manuel critique. Éditions Hors d’atteinte.

Les écrivains de Calais. (2020). Les voix de la " Jungle». Histoires de réfugiés au camp de Calais. La Guillotine.

Maldidier, D. (1990). L'inquiétude du discours. Textes de Michel Pêcheux. Éditions des cendres.

Mathieu, L. (1999). Une mobilisation improbable: l'occupation de l'église Saint-Nizier par les prostituées lyonnaises. Revue française de sociologie, 40(3), 475-99.

Noiriel, G. (2018). Une histoire populaire de la France. Agone.

Paveau, M. -A. (2017). Le discours des vulnérables. Proposition théorique et politique. Cadernos de Linguagem e Sociedade, 18(1), 135-57.

Però, D. et Solomos, J. (2010). Introduction: Migrant politics and mobilization: Exclusion, engagements, incorporation. Ethnic and Racial Studies, 33(1), 1-18.

Pette, M. (2019). Heurs et malheurs de la cause des migrants à Calais (1994-2016). Dans A. Lendaro (éd.), La crise de l'accueil (p. 209-230). La Découverte. https://www.cairn.info/la-crise-de-laccueil--9782348042843-p-209.htm. Consulté le 15 février 2021.

Olivier de Sardan, J. -P. (1998). Émique. Homme, 38(147), 151-66.

Spivak, G. C. (2010). Can the subaltern speak? Dans R. Morris (éd.), Can the Subaltern Speak?: Reflections on the history of an idea (p. 23-78). Columbia University Press.

Talpin, J. (2016a). Community organizing. De l'émeute à l'alliance des classes populaires aux États-Unis. Raisons d'agir.

Talpin, J. (2016b). La représentation comme performance. Le travail d'incarnation des classes populaires au sein de deux organisations communautaires à Los Angeles, USA. Revue française de science politique, 66(1), 91-115.

Telep, S. (2018). « Moi je whitise jamais. » Accent, subjectivité et processus d'accommodation langagière en contexte migratoire et postcolonial. Langage et société, 165(3), 31-49.

Tarragoni, F. (2014). De la personne au sujet politique. Une ethnographie des prises de parole populaires dans les assemblées de barrio au Venezuela. Participations, 9(2), 149-75.

Veniard, M. (2019). Le choix des mots : une forme de lutte à part entière. De Facto, journal de l'Institut des Migrations, 3 [En ligne]. http://icmigrations.fr/2019/01/15/defacto-3-001/

Veniard, M. (à paraître en 2022). « Ne pas parler à la place des premiers concernés » : Questionnements méthodologiques autour de la variation dialogique d'un impératif langagier dans le milieu des militants pour les droits des étrangers en France. Glottopol 36. 
Veniard, Marie. « 'Ne pas parler à la place des premiers concernés'. Étude d'une pratique politique d'éthique langagière chez des militants politiques pour les droits des étrangers en France. » Nouvelle Revue Synergies Canada, № 15 (2022).

Veron, D. (2019). «Sans combat, il n'y aura rien du tout!». L'engagement des sans-papiers pour leur régularisation (région parisienne, 2008-2011). Critique internationale, 84(3), 23-41.

Voirol, O. (2005). Les luttes pour la visibilité. Réseaux, 129-130(1), 2005, 89-121. 
Veniard, Marie. « 'Ne pas parler à la place des premiers concernés'. Étude d'une pratique politique d'éthique langagière chez des militants politiques pour les droits des étrangers en France. » Nouvelle Revue Synergies Canada, № 15 (2022).

\section{Annexe}

Le Torchon Brûle $\mathrm{n}^{\circ} 1$, journal édité par le Mouvement de libération des femmes (MLF) entre mai 1971 et juin 1973.

\section{La prise de parole}

Vous ne m'avez jamais donné la parole.

Vous ne m'avez jamais écoutée.

Vous me disiez à chaque fois : * Tu as l'air très seule, tu as besoin de parler avec quelqu'un, de communiquer ". Alors j'étais pleine d'espoir je me C:-ais ce coup-ci ça va marcher le dialogue, la vie a deux et tout... Mais la parole vous ne me la donniez pas. Ça ne vous intéressait pas de m'écou. ter. Vous me preniez et je me laissais prendre. Je me donnais même, maintenant j'ai compris, la parole je la prends, et je vois bien que vous ne m'écoutez pas. Vous essayez par tous les moyens de réduire ce que je dis, de l'envoyer ailleurs, je suis une enfant ou alors vous me regardex. comme un objet, et vous en profitez pour ne pas m'écouter. Parce que ce que je dis n'est pas ce que vous aviez envie d'entendre. C'est pour cela que vous ne me donniez pas la parole, parce que mon discours, vous aviez décidé, vous, de ce qu'il serait, vous l'aviez fait dans votre tête, vous n'aviez pas besoin de m'écouter.

Mais j'ai compris, la parole est une chose qui se prend.

Ca ne m'intéresse plus d'exister par rapport aux hommes, d'exister en face d'eux, d'être reconnue par eux en tant qu'être humain car j'ai compris qu'ils ne me reconnaitront jamais comme un être humain, qu'ils me réduiront toujours a n'ètre que l'objet de leur désir ou la compagne de leurs projets et de leur vie : femme-objet ou femme-enfant c'est le seul choix.

Désormais c'est face aux Femmes, c'est par rapport à elles que je veux exister. C'est là-bas seulement, c'est par eiles seulement que je peux êture reeonnue comme étre humain et c'est face à elles seuiement que ça m'intéresse d'exister.

Le mouvement, moi je ne fais plus que ça toute la journéc. Je vais de réunion en réunion et quand je ne suis pas en réunion, je vis ma condition de femme 24 heures sur 24 .

Le mouvement, moi je ne fais plus que ça toute la journce. 